\title{
Characterization of 3-strap antennas in ASDEX Upgrade
}

\author{
Volodymyr Bobkov ${ }^{1,}{ }^{*}$, Roberto Bilato ${ }^{1}$, Laurent Colas $^{2}$, Ralph Dux ${ }^{1}$, Eric Faudot $^{3}$, Helmut Faugel $^{1}$, Helmut Fünfgelder ${ }^{1}$, \\ Albrecht Herrmann ${ }^{1}$, Jonathan Jacquot ${ }^{1}$, Arne Kallenbach ${ }^{1}$, Daniele Milanesio ${ }^{4}$, Riccardo Maggiora ${ }^{4}$, Rudolf Neu $^{1,5}$, \\ Jean-Marie Noterdaeme ${ }^{1,6}$, Roman Ochoukov ${ }^{1}$, Thomas Pütterich ${ }^{1}$, Wouter Tierens ${ }^{1}$, Wei Zhang ${ }^{1,6}$, the ASDEX Upgrade \\ Team and the EUROfusion MST1 Team** \\ ${ }^{1}$ Max-Planck-Institut für Plasmaphysik, Boltzmannstr. 2, 85748 Garching, Germany \\ ${ }^{2}$ IRFM, CEA Cadarache, 13108 Saint Paul lez Durance, France \\ ${ }^{3}$ IJL UMR 7198, U. de Lorraine P2M, Fac. Des Sciences, BP 70239, F-54506 Vandœuvre Cedex, France \\ ${ }^{4}$ Politecnico di Torino, Torino, Italy \\ ${ }^{5}$ Technische Universität München, Boltzmannstr. 15, 85748 Garching, Germany \\ ${ }^{6}$ Applied Physics Department, University of Ghent, B-9000 Ghent, Belgium
}

\begin{abstract}
The response of the local RF current measured at limiters of 3-strap ICRF antenna to variations of power balance and phasing at $f_{\mathrm{ICRF}}=30 \mathrm{MHz}$ agrees qualitatively well with EM calculations by TOPICA and RAPLICASOL codes. Measurements of tungsten sputtering yield and DC current at the limiters correlate strongly with the local RF current. In contrast to findings for the 2-strap antennas, values of DC current are predominantly positive, and negative only for some locations and feeding parameters. Explanations can involve more physical mechanisms than only parallel sheath dynamics.
\end{abstract}

\section{Introduction}

Tungsten (W) production due to Ion Cyclotron Range of Frequencies (ICRF) power was drastically reduced by installation and proper feeding of the so-called 3-strap antennas in ASDEX Upgrade (AUG) [1]. Control of the RF image current pattern at the antenna limiters, monitored by local RF probe measurements, was crucial to minimize the ICRF-specific $\mathrm{W}$ sources. The measurements were also found to be in a qualitative agreement with electromagnetic (EM) calculations of local near-field at the antenna limiters by TOPICA [2]. This confirmed strong evanescence of the slow wave in the typical AUG conditions of the far scrape-off layer plasma [3]. It also proved the relevance of the approach to optimize the antenna design by lowering local RF currents and RF electrical field at the antenna limiters [4]. Recent experiments in Alcator C-Mod [5] have confirmed that conditions with reduced RF image currents correspond to reduced impurity release and reduced RF-induced plasma potentials.

In this work, we extend the characterization of local quantities in the conditions close to the RF image current cancellation at the limiters of the 3-strap antennas in AUG [1,2], both experimentally (section 1) and theoretically (section 2).

\section{Experimental characterization}

In [2], experimental characteristics were studied for the case of $f_{\text {ICRF }}=36.5 \mathrm{MHz}$. Here we discuss the responses of measurements to variations of strap phasing and power balance of the 3 -strap antenna for $f_{\mathrm{ICRF}}=30 \mathrm{MHz}$. This frequency is more convenient for EM modelling, as explained in section 3 .

The experimental conditions are similar to those described in [2]: ELM mitigated H-mode plasma at high density, with $\mathrm{P}_{\mathrm{ICRF}}=1 \mathrm{MW}$ with $\mathrm{H}$-minority heating and $\mathrm{P}_{\mathrm{NBI}}=5 \mathrm{MW}$, but at $B_{\mathrm{t}}=2.0 \mathrm{~T}$ in discharge \#33105. Figure 1 shows the 3 -strap antenna $4(a 4)$ in sector 12 of AUG and the locations of the following measurements: RF current (expressed as $50 \mathrm{Ohm}$ equivalent voltage $V_{\mathrm{RF}}$ ), DC current $I_{\mathrm{DC}}$ at limiter tiles numbered 01 to 12 , as well as spectroscopic measurements of $\mathrm{W}$ sputtering yield $Y_{\mathrm{W}}$ close to limiter tiles 01 to 03 and 07 to 09 . Figures 2 and 3 presents $V_{\mathrm{RF}}$ and $I_{\mathrm{DC}}$ expressed as $2 \mathrm{D}$ functions of the strap power balance $P_{\text {central }} / P_{\text {outer }}$ (where $P_{\text {central }}$ is the power to central strap and $P_{\text {outer }}$ is the sum of the power to the outer straps) and of the deviation of phasing between the central strap and the in-phase outer straps from dipole $\Delta \Phi$, for left and right antenna sides, respectively. Figure 4 shows $Y_{\mathrm{W}}$.

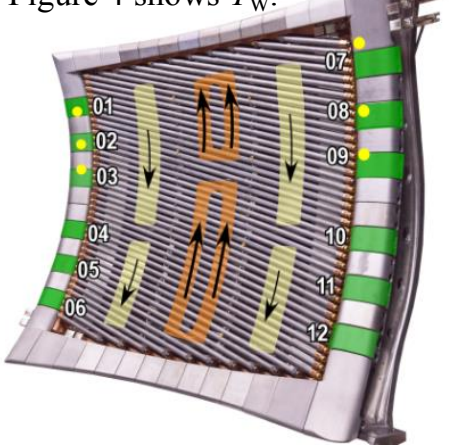

Fig. 1. 3-strap antenna with strap RF currents and measurement locations highlighted. 


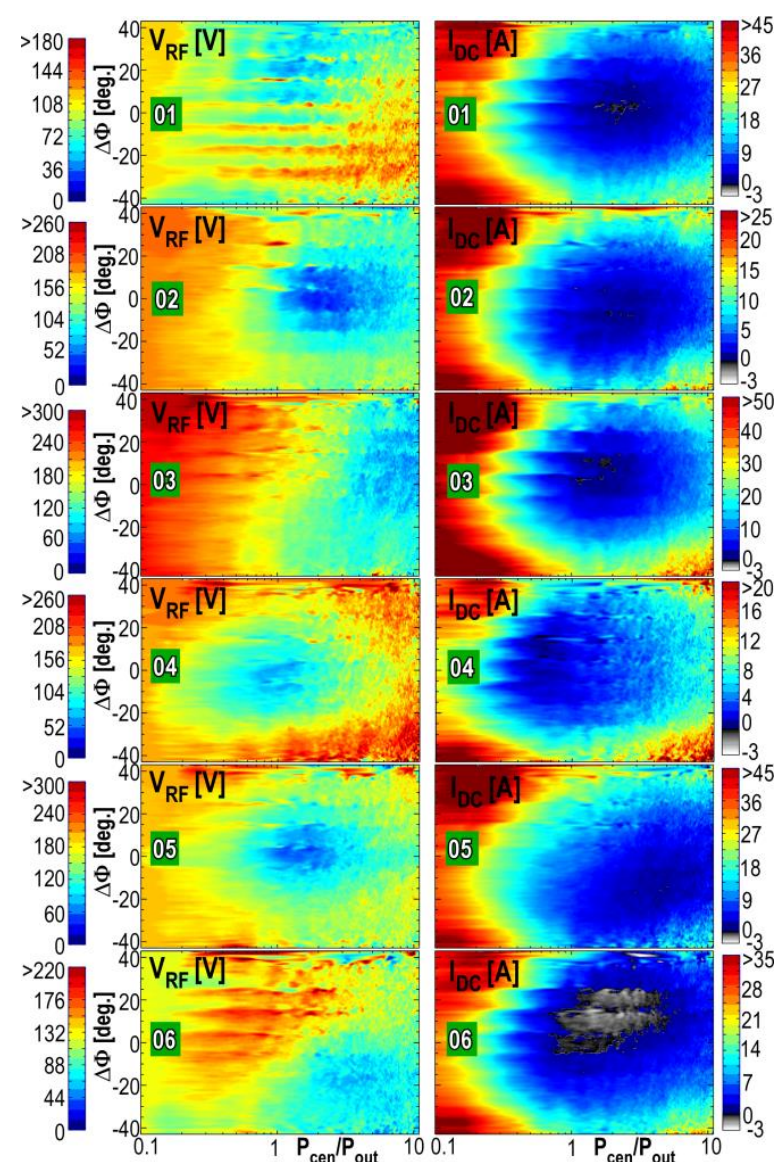

Fig. 2. $V_{\mathrm{RF}}, I_{\mathrm{DC}}$ as functions of $P_{\text {central }} / P_{\text {outer }}$ and $\Delta \Phi$ at various locations at the left antenna side (note different colour scales).

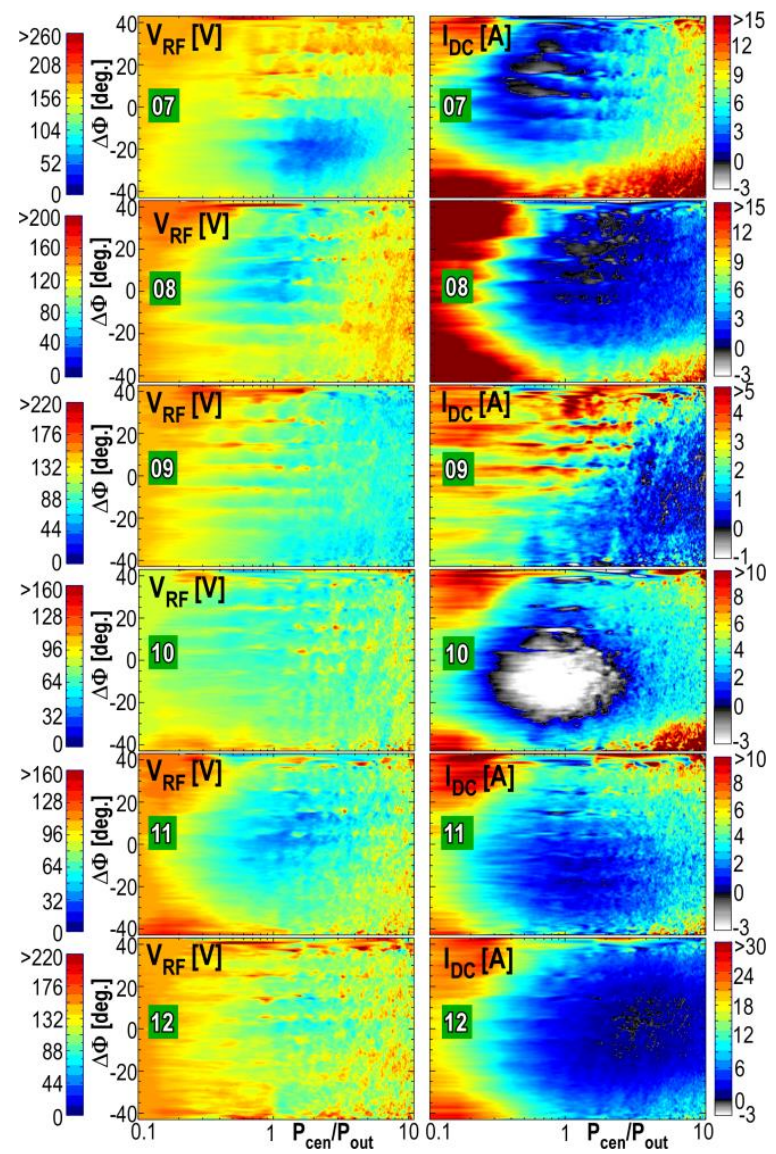

Fig. 3. $V_{\mathrm{RF}}, I_{\mathrm{DC}}$ as functions of $P_{\text {central }} / P_{\text {outer }}$ and $\Delta \Phi$ at various locations at the right antenna side (note different colour scales)
Although $V_{\mathrm{RF}}$ measurements at $f_{\mathrm{ICRF}}=30 \mathrm{MHz}$ react less sensitively to changes of $P_{\text {central }} / P_{\text {outer }}$ and of $\Delta \Phi$ than those in the case of $f_{\mathrm{ICRF}}=36.5 \mathrm{MHz}$ in [2], the main characteristics of the measurements remain similar. Clear location-dependent areas with minima of $V_{\mathrm{RF}}$ exist in the 2D strap power balance - phasing space. These areas indicate the best parameters for local RF image current cancellation. Horizontal stripes on $V_{\mathrm{RF}}$ are visible in Fig. 2 and Fig.3 and can be explained by evolving conditions during scans of $P_{\text {central }} / P_{\text {outer }}$ conducted at fixed $\Delta \Phi$, as well as by associated non-linearities. The $3 \mathrm{D}$ density profile in front of the antenna is likely evolving and affecting the overall balance of RF image currents at the antenna [2], as shown by calculations in [6]. Measurements of DC current $I_{\mathrm{DC}}$ have not been reported before for the 3-strap antennas and require a particular attention.

Previously, the DC measurements were described for some of the locations of the 2-strap antennas [7] in standard dipole phasing. Values of $I_{\mathrm{DC}}$ were predominantly negative on active antennas and positive on non-active antennas. However, these currents are predominantly positive for the active 3-strap antenna close to dipole phasing, as is shown in Fig. 2 and Fig. 3. Only several of the locations, such as $06,07,08$ and 10 have large areas with negative $I_{\mathrm{DC}}$. The areas of negative $I_{\mathrm{DC}}$ are enclosed in the areas with $I_{\mathrm{DC}}=0$, in many cases corresponding approximately to the minimum of $V_{\mathrm{RF}}$. However, crossing the condition $I_{\mathrm{DC}}=0$ could point to a change of dominant mechanism for collection of net DC current on the relatively large limiter tile, rather than to an optimized parameters range with a minimum of $\mathrm{W}$ sputtering. It is therefore questionable if the $I_{\mathrm{DC}}$ signals can be used for optimization of the antenna feeding parameters, although these signals have the highest sensitivity to those.

The observations indicate that the DC circuit for the 3 -strap antenna differs from that of the 2-strap antenna. Among the physical mechanisms deemed responsible for DC current collection, two are known to play a role: a) sheath rectification along the field lines, i.e. parallel sheath dynamics, modelled with such codes as SSWICH [8]; b) collection of ion current transverse to the magnetic field, which is observed in small RF plasma experiments with well-defined electrode geometry, such as Aline [9]. The second mechanism is not included in SSWICH and can be a key to explaining the positive $I_{\mathrm{DC}}$. It is also enhanced by perpendicular RF electric field at the limiters. If small experiments [9] can prove the importance of the second mechanism, the transverse components of RF electric field should be considered in addition to the parallel component for sheath rectification and $\mathrm{W}$ sputtering. Luckily for the antenna design optimizations, the transverse field components in front of the limiters are also decreased when the RF currents at the limiters are decreased.

As for the tungsten sputtering characterized by $Y_{\mathrm{W}}$ presented in Fig.4, it reacts to the feeding scans approximately in the same manner as $V_{\mathrm{RF}}$ in the corresponding locations, i.e. showing strong correlation with $V_{\mathrm{RF}}$ and $I_{\mathrm{DC}}$. The overall picture is very similar to that presented in [2] where $f_{\mathrm{ICRF}}=36.5 \mathrm{MHz}$. 

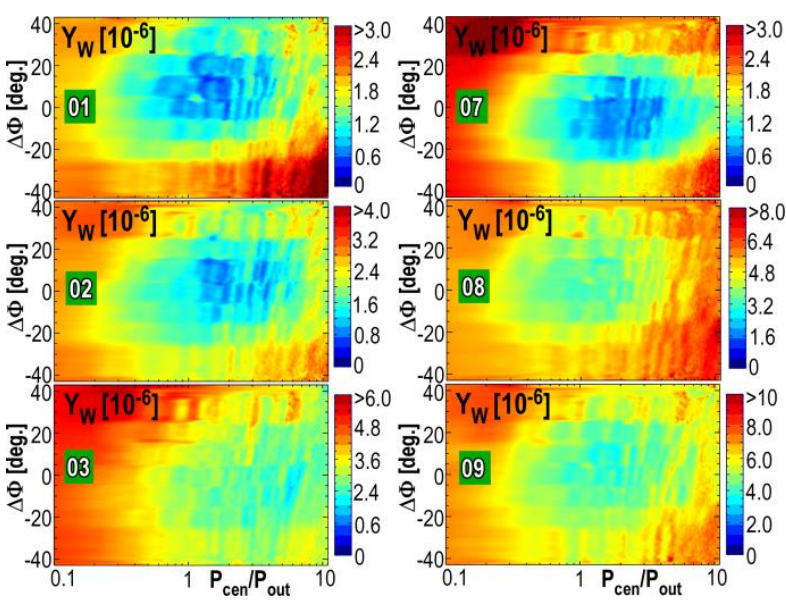

Fig. 4. $Y_{\mathrm{w}}$, as a function of $P_{\text {central }} / P_{\text {outer }}$ and $\Delta \Phi$ at locations 01 02,03 at the left and 07,08,09 at the right antenna side (note different colour scales).

\section{Theoretical characterization}

The case with $f_{\mathrm{ICRF}}=30 \mathrm{MHz}$ is better suited for modelling with EM codes, because the frequency is significantly lower than the resonant frequency of the straps, in particular that of the outer ones. Depending on model and plasma conditions, the latter is in the range between 36.5 and $36.9 \mathrm{MHz}$. Crossing this resonance frequency does not affect the near-field distribution significantly However working at $f_{\text {ICRF }}=30 \mathrm{MHz}$ helps to resolve issues in calculations with the RAPLICASOL [10] code used in this work.

We consider three cases modeled with EM codes, all using the same set of plasma profiles corresponding to the scenario of discharges \#31515 and \#33105. The first one uses a flat model of the 3-strap antenna in TOPICA as in [2]. The second one introduces a more realistic model of the antenna in curved geometry in TOPICA [11]. The third case applies the same flat geometry as in the first case, but in RAPLICASOL code.
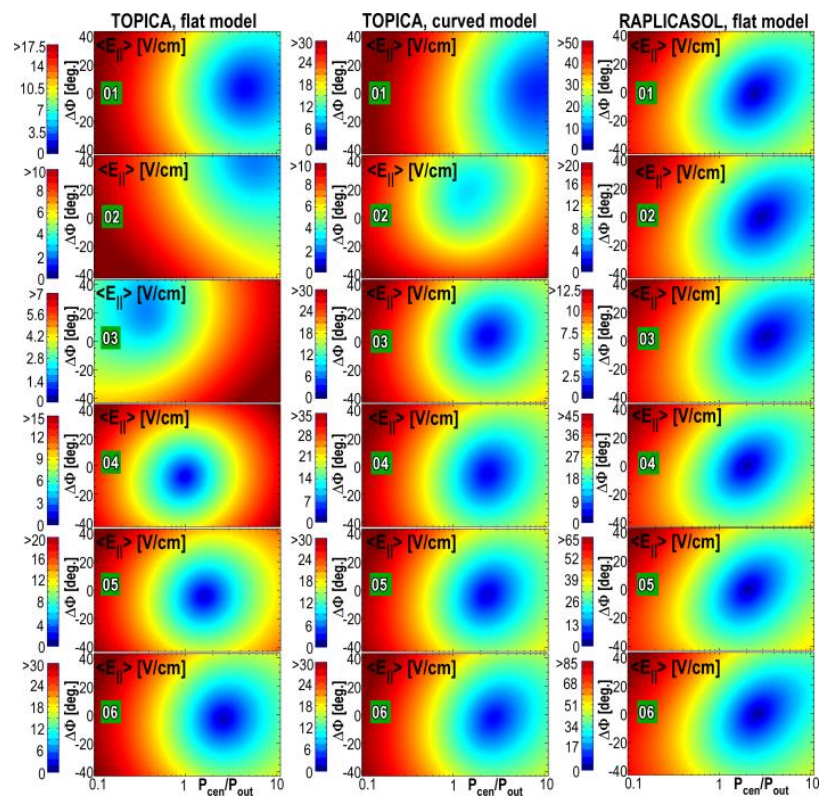

Fig. 5. $\left\langle E_{\|}\right\rangle$calculated with TOPICA flat (left), TOPICA curved (middle) and RAPLICASOL flat (right) models at the left antenna side locations (note different colour scales).
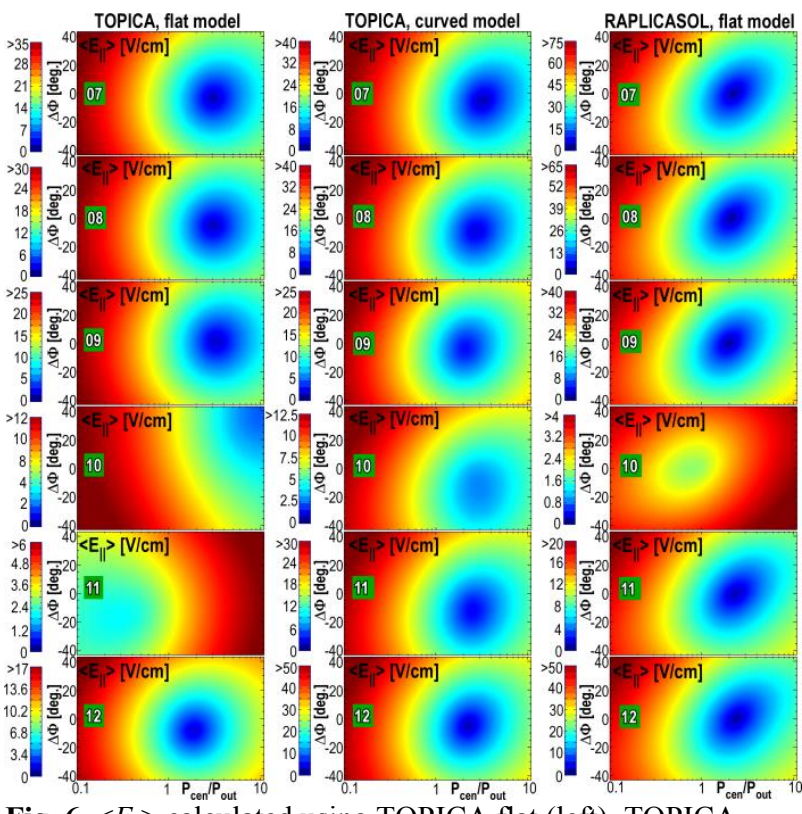

Fig. 6. $\left\langle E_{\|}>\right.$calculated using TOPICA flat (left), TOPICA curved (middle) and RAPLICASOL flat (right) models at the right antenna side locations (note different colour scales).
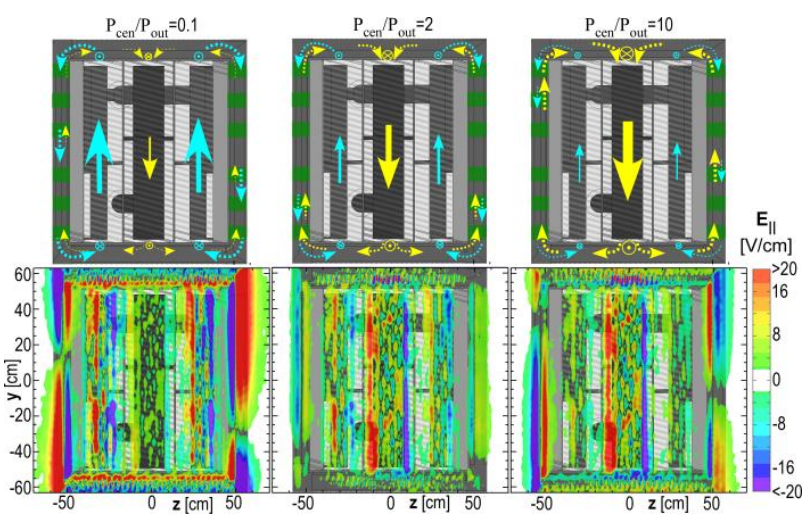

Fig. 7. $E_{\|}$for flat TOPICA model for different cases of power balance in dipole for $30 \mathrm{MHz}$, with graphics showing RF current circulation at limiters (top). Purely vertical dashed arrows on the limiters indicate locations of sign reversal of parallel component of RF current.

As a theoretical "proxy", we use spatially averaged values of the $E_{\|}$field $\left\langle E_{\|}>\right.$calculated just in front of the antenna limiters at locations 01-12. Firstly, due to strong evanescence of the slow wave, $\left\langle E_{\|}\right\rangle$is the main contributor to the RF sheath driving voltage and rectified sheath voltage, as has been discussed in [2] and has been shown theoretically in $[3,12]$. Secondly, this quantity is primarily a linear function of local RF current (in particular, of its parallel component) at fixed geometrical and loading conditions. Thirdly, $\left\langle E_{\|}\right\rangle$is an engineering quantity which can easily be used for antenna optimization with practically any EM code. The ICRF power is scaled to $500 \mathrm{~kW}$ for all the simulations.

Figures 5 and 6 show the $\left\langle E_{\|}\right\rangle$calculations in the three different modelling cases (columns) for the left and the right antenna sides, respectively. Location-dependent areas with minima close to the dipole phasing and to $P_{\text {central }} / P_{\text {outer }} \approx 2$ are well reproduced in all three modelling cases compared to the experimental $V_{\mathrm{RF}}$ diagrams, with details which differ. These minima correspond to the conditions of the local cancellation of all components of 
RF current. Spatial $E_{\|}$distribution is shown in Fig. 7 for $P_{\text {central }} / P_{\text {outer }}$ of $0.1,2.0$ and 10.0 in dipole phasing calculated for flat model in TOPICA, together with qualitative presentation of the RF image current circulation on the limiters.

According to Fig.5 and Fig.6, minima of $\left\langle E_{\|}\right\rangle$ become broader somewhat above the midplane for the left side (locations 02, 03) and somewhat below the midplane on the right side (locations 10, 11). In experiment, broader regions with minima of $V_{\mathrm{RF}}$ are also observed on the right side at about the midplane (locations 09, 10 in Fig. 3). Close to the regions with broader minima, $\left\langle E_{\|}\right\rangle$are generally lower, according to the calculations. Here, parallel projection of the RF image current of individual straps, flowing on the limiters, changes sign (unbalanced cases in Fig.7), as well as the total RF current at the limiters is reduced. This results in minima at the $E_{\|}$maps. However in the experiment, there is no clear tendency for significantly lower values in the locations corresponding to these.

The more detailed model with curved geometry in TOPICA produces coupling parameters which match experimental values significantly better [11]. Consequently, a more realistic relation between the power balance and the voltage balance inside the antenna (presented in [1]), is reproduced and the confidence in results using the curved antenna model is higher.

Although the general trends of the local quantities are well described by the simulations, and one can speak of at least a qualitative agreement; quantitative one-to-one correspondence between the experiments and the calculations is difficult due to existing limitations and uncertainties. As can be inferred from [6], details of 3D density profile have a significant effect on the RF image currents and $E_{\|}$at the limiters. On top of that, the profiles are not stationary in experiments [2]. Even if a better qualitative correspondence were found by refining experimental input and by detailing the models further, the variety of the profiles in the experiment would make it very challenging to optimize antenna design further, in order to have minima at the same values of $P_{\text {central }} / P_{\text {outer }}$ and $\Delta \Phi$ at all limiter locations, and this for a broad variety of plasma conditions.

One of alternative approaches to optimize the 3-strap antenna further in experiments is increasing the number of actuators by making the antenna limiters active, fed independently by feedback-controlled RF sources. This could allow counteracting the residual RF currents at the limiters by destructive interference. However this approach needs testing on small-scale machines. Another actuator which already is available in the experiments and planned to be used in the future to tackle RF fields in the scrape-off layer is the phasing between antenna pairs.

\section{Summary}

Experimental local values of RF current, DC current, W sputtering yield were presented for multiple locations at the 3-strap antenna as functions of variations of antenna strap power balance and strap phasing. The quantities experience a minimum close to dipole phasing when power of the central strap is close to the double of the sum of the power to the outer straps.

The DC current can however reverse sign and is predominantly positive in contrast to previous studies with 2-strap antennas [7]. This implies a complicated nature of the DC circuit, which can also involve a collection of ion current transverse to the magnetic field.

Comparison of measured RF current at the limiters with EM calculations using a flat antenna model in TOPICA and RAPLICASOL as well as a more realistic curved antenna model in TOPICA shows that qualitative features of the experimental observations are captured by the calculations. Better quantitative agreement between simulations and measurements is presenting a challenging task, given the uncertainties and variations in the experimental input $[2,6]$. An independent method to reduce the RF currents by actively feeding the antenna limiters and synchronizing operation of all four antennas in ASDEX Upgrade could be tested in the future.

We refer to [13] where the recent progress in solving the problems of the antenna-plasma interface in ASDEX Upgrade is summarized.

This work has been carried out within the framework of the EUROfusion Consortium and has received funding from the Euratom research and training programme 2014-2018 under grant agreement No 633053. The views and opinions expressed herein do not necessarily reflect those of the European Commission.

\section{References}

1. V. Bobkov et al., Nucl. Fusion 56084001 (2016)

2. V. Bobkov et al., Plasma Phys. Control. Fusion 59014022 (2017)

3. L. Colas et al., Plasma Phys. Control. Fusion 59025014 (2017)

4. V. Bobkov et al., Nucl. Fusion 50035004 (2010)

5. S. Wukitch et al., "Towards ICRF Antennas Compatible with High Performance Plasmas: Characterization and Mitigation of ICRF Antenna - Plasma Edge Interaction", this conference, Inv-08

6. W. Tierens et al., "3-Dimensional density profiles in edge plasma simulations for ICRF heating", this conference, A-25

7. V. Bobkov et al., J. Nucl. Mater. 415 S1005 (2011)

8. J. Jacquot, Phys. Plasmas 21(6) 061509 (2014)

9. E. Faudot et al., this conference, "DC currents collected by a RF biased electrode quasi-parallel to the magnetic field", A-33

10. J. Jacquot et al., AIP Conf. Proc. 1689050009 (2015)

11. D. Milanesio et al., "Analysis of the ASDEX Upgrade 3strap antenna with TOPICA code: curved vs. flat 3D geometry", this conference, C-22.

12. W. Tierens et al., "Nonlinear plasma sheath potential in the ASDEX Upgrade 3-strap antenna: a parameter scan", submitted to Nucl. Fusion

13. W. Zhang, "Recent progress on improving ICRF coupling and reducing rf-specific impurities in ASDEX Upgrade", this conference, Inv-07 